\title{
O ICMS ecológico como instrumento econômico de melhorias ambientais e sociais em alguns municípios mineiros
}

Flora Magdaline Benitez Romero ${ }^{1}$

Liniker Fernandes da Silva ${ }^{2}$

Crismeire Isbaex ${ }^{3}$

Eliana Boaventura Bernardes Moura Alves ${ }^{4}$

Laércio Antônio Gonçalves Jacovine ${ }^{5}$

Márcio Lopes da Silva ${ }^{6}$

\section{Resumo}

O enfoque da conservação e manutenção dos recursos naturais, assim como os mecanismos de reconhecimento e compensação, tem ganhado aceitação no ambiente de políticas públicas. Uma ação que demonstra isso é a criação do Imposto sobre Circulação de Mercadorias e Prestação de Serviços Ecológico (ICMS Ecológico). Partindo-se do pressuposto de que vários municípios têm se aproveitado desse instrumento para aumentar suas receitas, sem aplicar corretamente esses recursos em medidas de preservação ambiental, o objetivo geral deste trabalho foi analisar a relação entre as variáveis socioeconômicas e ambientais com o montante de ICMS ecológico repassado pelo estado e recebido pelos municípios. Para tanto, dez municípios do estado de Minas Gerais foram selecionados e tiveram seus dados analisados durante o período de 2005-2010. As variáveis consideradas para análise foram: Investimento em Saneamento, Gestão Ambiental, População, Receita Total e Índice FIRJAN de Desenvolvimento Municipal - IFDM. Para verificar as relações entre as variáveis foi utilizada a análise de componentes principais (PCA), que é uma técnica matemática da análise multivariada. Os resultados mostraram que o ICMS Ecológico não tem correlação com a aplicação de recursos em gestão ambiental e saneamento, bem como não apresenta relação com o desenvolvimento social dos municípios avaliados. O município de Extrema possui a menor arrecadação de ICMS Ecológico e, ao mesmo tempo, os maiores índices de desenvolvimento social e investimentos em gestão ambiental. Desta forma, conclui-se que a maior arrecadação do ICMS Ecológico não se traduz em maiores investimentos em saneamento e gestão ambiental, tampouco melhorias sociais.

Palavras-chave: Redistribuição de recursos. Qualidade de vida. Serviços ambientais.

1 Universidade Federal de Viçosa - UFV, doutoranda em Ciências Florestais. magdalyne@hotmail.com.

2 Universidade Federal de Viçosa - UFV, doutorando em Ciências Florestais. linikerfs@gmail.com.

3 Universidade Federal de Viçosa - UFV, doutoranda em Ciências Florestais. engisbaex@gmail.com.

4 Universidade Federal de Viçosa - UFV, doutoranda em Ciências Florestais. elianabbm@gmail.com.

5 Universidade Federal de Viçosa - UFV, professor do Departamento de Engenharia Florestal. jacovine@ufv.br.

6 Universidade Federal de Viçosa - UFV, professor do Departamento de Engenharia Florestal. mlopes.ufv@gmail.com. 


\section{Introdução}

Os recursos naturais renováveis (RN) são considerados bens livres existentes na natureza em quantidade ilimitada ou superior à quantidade necessária para satisfazer as necessidades do homem. Dessa forma, o ser humano explora os recursos naturais renováveis sem a preocupação de que esses bens possam se tornar escassos, não contemplando a possibilidade do seu esgotamento. No entanto, tal comportamento pode trazer problemas e danos ambientais que futuramente não poderão ser resolvidos, mesmo com recursos financeiros disponíveis (MAY et al., 2003; BERKES, 2005).

Como forma de avaliar esses danos, a economia estuda as relações produtivas e a alocação dos recursos produtivos escassos entre as atividades competitivas, com o objetivo de gerar bem-estar ao homem. A nova alternativa implantada e incorporada à economia é a introdução, dentro do sistema linear, do meio ambiente como assimilador de resíduos e gerador de utilidade ao homem de forma direta. Afinal, o sistema linear considera o meio ambiente como apenas fornecedor de matéria-prima, o que gera algumas distorções e falhas nos mercados, tornando o sistema ineficiente para alocar os recursos arrecadados, provocando externalidades (PEARCE; TURNER, 1990; PINHEIRO et al., 2010).

A presença de externalidades também contribui para explicar porque os mercados privados são ineficientes para alocar os recursos. Externalidades ocorrem quando o consumo e/ou a produção de um determinado bem afetam os consumidores e/ou produtores, em outros mercados, e esses impactos não são considerados no preço de mercado do bem em questão, podendo ser positivas (benefícios externos) ou negativas (custos externos). Tais distorções geram ineficiências tanto na produção quanto no consumo. Deste modo, é necessário implementar mecanismos capazes de corrigir ou valorizar tais externalidades (PEARCE; TURNER, 1990; REZENDE; OLIVEIRA, 2001).

Uma das formas de comando e controle do governo é a taxação por meio do Imposto sobre Circulação de Mercadorias e Prestação de Serviços (ICMS). A Constituição Federal promulgada em 1988, em seu artigo 145, trata da Tributação e do Orçamento e classifica as espécies de tributos em impostos, taxas e contribuições de melhoria. 0 artigo 158, inciso IV da Constituição, trouxe alterações para a dinâmica de funcionamento do Sistema Tributário Brasileiro, ao tratar da "Repartição das Receitas Tributárias", estabelecendo que $25 \%$ do ICMS arrecadado pelo estado pertence aos municípios (BRASIL, 1988).

Em Minas Gerais, o ICMS Ecológico foi implantado em 1995, a partir da Lei n. ${ }^{0}$ 12.040/95 (Lei Robin Hood), com vigência pela Lei n 18.030/2009 (MINAS GERAIS, 2009), e tem como objetivo reduzir as diferenças econômicas e sociais entre os municípios e incentivar a aplicação de recursos na área social (BENSUSAN, 2002). O ICMS é um imposto estadual que está embutido no preço das mercadorias e serviços, sendo relativo às atividades de circulação, de transporte interestadual e intermunicipal, de energia e de comunicação.

A distribuição de ICMS Ecológico em Minas Gerais até 2009 era feita conforme os critérios de área geográfica, população, população dos 50 municípios mais populosos, educação, saúde, meio ambiente, patrimônio cultural, produção de alimentos e receita própria (MINAS GERAIS, 2009). Assim, os municípios de médio e grande porte estavam entre os que mais recebiam o imposto ecológico (MINAS GERAIS; 2009; MASSOTE; VIEIRA, 2014). Após a nova legislação, os repasses do ICMS aos municípios estão sendo feitos conforme critérios de educação, produção de alimentos, meio ambiente, saúde, recursos hídricos, municípios sede de estabelecimentos penitenciários, esporte, turismo, ICMS Solidário e mínimo per capta (MINAS GERAIS, 2009).

Diante do princípio do Protetor-Recebedor, 1,1\% do ICMS é repassado aos municípios, sendo que a distribuição desse montante é realizada em função do Índice de Meio Ambiente (IMA). O IMA 
é composto por três subcritérios, ponderados pelos respectivos pesos, a saber: Índice de Conservação (IC - 45,45\%), referente às Unidades de Conservação e outras áreas protegidas; Índice de Saneamento Ambiental (ISA 45,45\%), referente aos aterros sanitários, estações de tratamento de esgotos e usinas de compostagem; mais recentemente, Índice de Mata Seca (IMS - 9,1\%), referente à presença e proporção em área da fitofisionomia Mata Seca no município (SECRETARIA DE ESTADO DE MEIO AMBIENTE E DESENVOLVIMENTO SUSTENTÁVEL - SEMAD, 2016). Neste sentido, o ICMS Ecológico é destinado a compensar e estimular a conservação e o uso sustentável dos recursos ambientais (FRANCO; FIGUEREDO, 2007; FERNANDES et al., 2011).

Partindo-se do pressuposto de que o repasse do ICMS Ecológico não está sendo aplicado de maneira coerente na adoção de medidas de preservação e que ele pode se tornar um instrumento econômico para o controle ambiental no estado, objetivou-se com este trabalho analisar a relação entre as variáveis socioeconômicas e ambientais com o montante de ICMS ecológico repassado pelo estado e recebido pelos municípios. Para tanto, dez municípios do estado de Minas Gerais foram selecionados e tiveram seus dados analisados durante o período de 2005 - 2010.

\section{Material e métodos}

\section{Definição dos municípios e banco de dados}

Dos 853 municípios mineiros existentes, foram selecionados os dez que apresentaram a maior arrecadação do ICMS Ecológico: Marliéria, Timóteo, Extrema, São João das Missões, Gonçalves, São João Batista do Glória, Toledo, Camanducaia, Sapucaí-Mirim e Itapeva (MASSOTE e VIEIRA, 2014; FUNDAÇÃO JOÃO PINHEIRO, 2013). Os municípios foram selecionados em virtude de sua representatividade no total de recursos recebidos pelos municípios mineiros (Tabela 1).

Tabela 1. ICMS ecológico recebido por todos os municípios de Minas Gerais e a representatividade dos municípios avaliados durante o período de 2005 a 2010

\begin{tabular}{c|cc} 
& $\begin{array}{c}\text { ICMS recebido por todos } \\
\text { municípios mineiros (R\$) }\end{array}$ & $\begin{array}{c}\text { Percentual recebido pelos } \\
\text { municípios avaliados }\end{array}$ \\
2005 & & 2,74 \\
2006 & & 3,68 \\
2007 & & 5,86 \\
2008 & & 6,69 \\
2009 & $55.328 .080,57$ & 6,24 \\
2010 & & 7,49 \\
\hline
\end{tabular}

Fonte: Elaborado pelos autores.

As variáveis analisadas foram: população, receita total, gastos da prefeitura com saneamento e em gestão ambiental, Índice FIRJAM de Desenvolvimento Municipal (IFDM) e os recursos recebidos pelos municípios por meio do ICMS ecológico. Essas variáveis representam alguns dos critérios para repartição do ICMS aos municípios mineiros, bem como dão indícios de como os municípios têm investido para melhoria do meio ambiente.

Os dados relativos ao ICMS Ecológico recebido pelos municípios avaliados foram obtidos no website do ICMS Ecológico - Brasil (THE NATURE CONSERVANCY - TNC, 2015). Os valores relativos à receita total, gastos em saneamento e gestão ambiental de cada município foram obtidos do website "Compara Brasil" (FRENTE NACIONAL DOS PREFEITOS, 2015). O IFDM (Índice FIRJAM 
de Desenvolvimento Municipal), disponibilizado pela Federação das Indústrias do Rio de Janeiro, foi utilizado como um indicador do desenvolvimento social dos municípios.

Os dados de população avaliados foram coletados no site do Instituto Brasileiro de Geografia e Estatística (IBGE, 2015). Foram disponibilizados os dados de população referentes aos anos de 2000, 2007 e 2010. Para que se tivessem as populações dos municípios em todo o intervalo entre 2004 e 2010, considerou-se que a mudança nos dados entre 2000 e 2007 e entre 2007 e 2010 seguem ritmo linear. Desta forma, interpolaram-se os dados obtendo a população dos municípios entre os anos de 2004 e 2010.

De posse das observações mencionadas, foi possível montar um banco de dados, por município, com todas as informações citadas, compreendendo uma série histórica entre 2005 e 2010.

\section{Análise dos dados}

Em função do número de variáveis estudadas, optou-se pelo uso da Análise de Componentes Principais (ACP) como ferramenta para avaliar os dados. Essa análise foi escolhida devido a sua característica de avaliação da correlação entre as variáveis.

A ACP reescreve os dados amostrados em um novo conjunto de eixos, denominados componentes principais, ortogonais entre si, cuja variância é decrescente da primeira para a última componente. Desta forma, a primeira componente principal detém maior representatividade das informações que a segunda componente e assim por diante. Tal procedimento reduz o número de informações que deve ser avaliado, facilitando o trabalho de interpretação (MAXWELL, 1977).

A análise ACP foi realizada com o pacote Vegan do Software R (OKSANEN et al., 2015) e os gráficos foram plotados com auxílio do programa Sigma Plot versão 11.0.

A análise dos componentes principais, referente aos 10 municípios foi obtida pela função Scores que determina a relação entre os municípios com as variáveis estudadas.

\section{Análise de Componentes Principais}

Um conjunto de dados de uma amostra com $n$ unidades e $p$ variáveis pode ser descrito matricialmente como:

$$
\mathrm{X}=[\mathrm{Xij}]
$$

em que $X_{i j}$ corresponde ao valor da j-ésima variável $(j=1, \ldots, p)$ para a $i$-ésima unidade amostral $(i=1, \ldots, n)$.

A análise de componentes principais transforma a matriz $X$ com $p$ variáveis em uma matriz $Y$ de $p$ variáveis hipotéticas não correlacionadas entre si, as quais decrescem em variância da primeira para a última (MAXWELL, 1977). A primeira coluna representa a primeira componente principal $Y_{i}$. Ela representa a soma ponderada das variáveis $X_{j}(j=1, \ldots, p)$, representada da seguinte forma:

$$
Y_{1}=X_{1} u_{11}+X_{2} u_{21}+\ldots+X_{p} u_{p l}
$$

em que $u_{j l}$ representa os pesos das $j$-ésimas variáveis na componente. Para a amostra com $n$ unidades, a notação matricial é:

$$
Y=X u
$$

em que, $Y$ é o vetor $n x 1$ de valores em $Y_{i}(i=1, \ldots, n)$ e $u$ representa o vetor $p x 1$ dos pesos a serem determinados.

A variância de $Y_{1}$ é uma porção da variância total, sendo a raiz característica $\lambda 1:\left(\left(\lambda_{1} / \sum \lambda_{i}\right)\right.$ $x 100(i=1, \ldots, n)$ 
Existem dois tipos de análise de componentes principais. Quando as variáveis analisadas apresentam unidades diferentes, caso deste estudo, ou apresentam variância muito discrepante, o aconselhável é utilizar a ACP de correlação. Neste caso, as variáveis são reduzidas. Tal procedimento ocorre antes de se iniciarem os passos descritos anteriormente.

\section{Resultados e discussão}

Os três primeiros componentes explicaram aproximadamente $87 \%$ da variação total dos dados analisados (Tabela 2). Segundo o critério da proporção, as componentes principais devem explicar determinada porcentagem da variabilidade dos dados. 0 padrão é que se utilizem as componentes que expliquem pelo menos 80 \% dos dados. Assim, deve-se utilizar os três primeiros componentes principais para a análise em questão visto que as duas primeiras componentes explicam apenas 73 $\%$ da variação dos dados (Tabela 2 ).

Os Loadings, relação entre as variáveis e os componentes principais, mostram que as variáveis Saneamento, Receita e População Municipal têm maior correlação com o componente principal 1 (Tabela 2). As variáveis IFDM e ICMS Ecológico têm maior relacionamento com o componente principal 2 e os investimentos com Gestão ambiental são mais relacionados ao Componente Principal 3.

Tabela 2. Loadings das componentes principais.

\begin{tabular}{ccccccc}
\hline \multirow{2}{*}{ Variável } & \multicolumn{7}{c}{ Componente Principal } \\
\cline { 2 - 7 } & 1 & 2 & 3 & 4 & 5 & 6 \\
\hline Receita & $-0,557$ & $-0,003$ & 0,019 & 0,240 & $-0,179$ & 0,774 \\
População & $-0,526$ & 0,006 & 0,025 & 0,459 & $-0,377$ & $-0,608$ \\
Saneamento & $-0,475$ & 0,232 & 0,297 & $-0,104$ & 0,777 & $-0,136$ \\
IFDM & $-0,272$ & $-0,579$ & 0,390 & $-0,614$ & $-0,237$ & $-0,072$ \\
ICMS Ecológico & $-0,275$ & 0,555 & $-0,454$ & $-0,586$ & $-0,250$ & $-0,062$ \\
Gestão & $-0,196$ & $-0,550$ & $-0,743$ & 0,016 & 0,322 & $-0,055$ \\
\hline Autovalores & 3,08 & 1,31 & 0,83 & 0,51 & 0,26 & 0,01 \\
Var. acumulativa (\%) & 0,514 & 0,732 & 0,870 & 0,955 & 0,998 & 1,000 \\
\hline
\end{tabular}

Fonte: Elaborado pelos autores.

O comportamento das variáveis em relação aos componentes principais pode ser visto de forma gráfica na Figura 1. O IFDM se diferenciou das demais variáveis analisadas, indicando que as demais variáveis estudadas não influenciam, de forma decisiva, a qualidade social. Os investimentos em gestão ambiental e saneamento não tiveram correlação com as variáveis estudadas.

A variável ICMS Ecológico também se diferenciou das demais, ou seja, não se pode considerar a correlação da referida variável com saneamento, receita, população e saneamento (Figura 1). Dessa forma, é possível afirmar que com maior recebimento de ICMS Ecológico, não existem garantias de investimentos na gestão ambiental e saneamento, bem como no desenvolvimento social. 
Figura 1. Gráfico do componente principal 1 versus componente principal 2 e componente principal 3

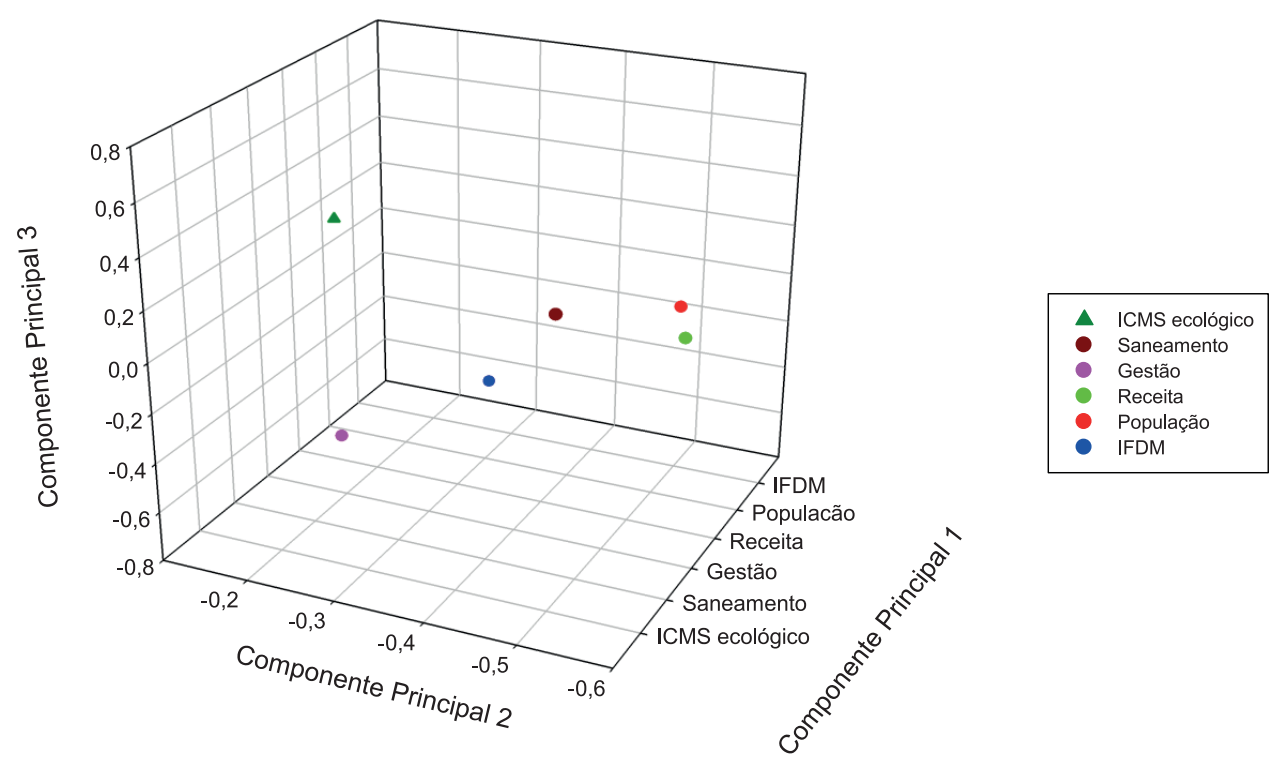

Fonte: Elaborado pelos autores.

A Figura 2 mostra a distribuição dos scores ao longo das componentes principais 1,2 e 3 . Observa-se que os municípios Marliéria e São João Batista da Glória apresentam scores semelhantes em todos os componentes e maior arrecadação de ICMS Ecológico. O município de Extrema possui a menor arrecadação de ICMS Ecológico e, ao mesmo tempo, os maiores índices de desenvolvimento social e investimentos em gestão ambiental. Os demais municípios apresentam, de forma geral, maior recebimento do ICMS Ecológico, entretanto, não estão tendo maior desenvolvimento social.

Figura 2. Scores dos municípios estudados, entre 2005 e 2010, na componente principal 1, versus a componente principal 2 e componente principal 3
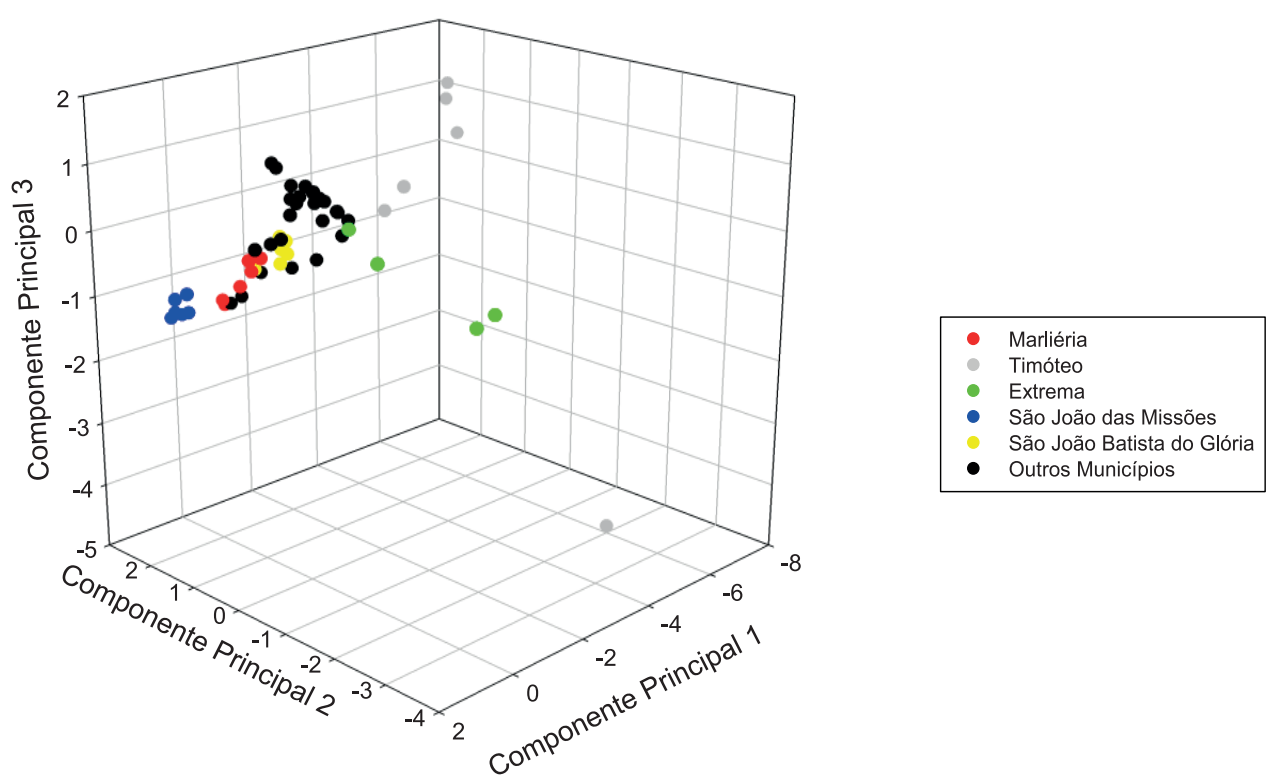

Fonte: Elaborado pelos autores.

Conforme Minas Gerais (2009), a distribuição do ICMS Ecológico é realizada com base nos critérios sociais e ambientais, como por exemplo, a presença de unidades de conservação (FERNANDES et al., 2011). Neste contexto, determinados municípios têm de se adequar para impossibilidade no 
uso econômico de áreas em função da introdução de áreas de preservação (MONTE; SILVA, 2009). Dessa forma, esperava-se que os municípios de maior arrecadação destes recursos tivessem condições sociais semelhantes aos demais, o que não ocorreu neste trabalho.

Uma hipótese que suporta o descompasso entre o recebimento do ICMS ecológico e o índice IFDM, bem como os investimentos em saneamento e gestão ambiental, é a ausência de regras definidas para aplicação deste recurso. Outra hipótese, apontada por Botelho (2007), é a de que o ICMS ecológico não remunera adequadamente os municípios por manterem suas áreas conservadas. Desta forma, os municípios não teriam recursos suficientes para os investimentos em questão. Uma alternativa seria o estado aumentar progressivamente o volume de recursos que remuneram os serviços ecológicos prestados pelos municípios (EUCLYDES, 2013).

Mesmo recebendo menores recursos do ICMS ecológico, o município de Extrema gerou melhor padrão social e maiores investimentos em gestão ambiental e saneamento, confirmando a preocupação que a governança local apresenta com as questões ambientais, também observada por Jardim e Bursztyn (2015). De acordo com os mesmos autores, o pagamento por serviços ambientais (PSA) na questão dos recursos hídricos está plenamente consolidado no município, contando com a colaboração de diversos atores da sociedade. Essa união em torno do PSA ajuda a explicar a posição de destaque que o município tem nesta área.

\title{
Conclusões
}

O recebimento de ICMS Ecológico dos dez municípios mineiros não tem como consequência direta maiores investimentos em saneamento e gestão ambiental, bem como no desenvolvimento social para a maioria dos municípios avaliados.

As normativas que regulam o ICMS em nível nacional dão liberdade aos estados para a aplicação e distribuição dos recursos. Em Minas Gerais não se estabelece especificamente a aplicação dos recursos do ICMS Ecológico para as atividades de saneamento, gestão ambiental e desenvolvimento social, que estão relacionadas diretamente à melhoria da qualidade de vida da população desses municípios.

\section{Ecological ICMS as economic instrument for environmental and social improvements in some municipalities of Minas Gerais state}

\begin{abstract}
The focus of conservation and maintenance of natural resources, as well as the mechanisms for recognition and compensation, have gained acceptance in the field of public policies. The creation of Tax on Circulation of Goods and Ecological Services Compensation (Ecological ICMS) demonstrates it. Assuming several municipalities have taken advantage of this tool to increase their revenue without properly applying these resources on environmental preservation measures, the aim of this study was to compare the relationship between socioeconomic and environmental variables to the amount of ecological ICMS passed by the state and received by municipalities. Therefore, ten municipalities from Minas Gerais state were selected and their data were analyzed during the period of 2005-2010. The variables considered for analysis were: Investment in Sanitation, Environmental Management, Population, Total Revenue and Index of Municipal Development FIRJAN - IFDM. To check the relationship
\end{abstract}


between variables, principal component analysis (PCA), which is a mathematical technique of multivariate analysis, was used. The results showed Ecological ICMS has no correlation with the application of environmental management and sanitation resources, and it is not related to the social development of the assessed municipalities. The Extrema municipality has the smallest revenue of Ecological ICMS and, at the same time, the highest rates of social development and investments in environmental management. Therefore, we conclude the largest revenue of Ecological ICMS does not translate into bigger investments in sanitation and environmental management, nor social improvements. Keywords: Reallocation of resources. Quality of life. Environmental services.

\section{Referências}

BENSUSAN, N. ICMS ecológico: um incentivo fiscal para a conservação da biodiversidade. In: BENSUSAN, N. (Org.). Seria melhor mandar ladrilhar? Biodiversidade como, para que, por quê. Brasília: Universidade de Brasília, 2002, p. 13-20.

BERKES, F. Sistemas sociais, sistemas ecológicos e direitos de apropriação de recursos naturais. In: VIEIRA, P. F.; BERKES, F.; SEIXAS, C. S. Gestão integrada e participativa de recursos naturais: conceitos, métodos e experiências. Florianópolis: Secco/APED, 2005. p.47-72.

BOTELHO, D. O. ICMS Ecológico como instrumento de política ambiental em Minas Gerais. 2007. 117 f. Dissertação (Mestrado em Administração). Universidade Federal de Lavras.

BRASIL. Constituição da República Federativa do Brasil de 1988. Disponível em: <http://www. planalto.gov.br/ccivil_03/Constituicao/Constituicao.htm>. Acesso em: 05 maio 2015.

EUCLYDES, A. C. P. Contradições da política ambiental por meio de incentivos financeiros: os casos do ICMS ecológico e da CFEM nos municípios do quadrilátero ferrífero (Minas Gerais, Brasil). Revista Árvore, Viçosa, v. 37, n. 6, p. 1083-1092. nov./dez. 2013. Disponível em: <http://www.scielo.br/ scielo.php?script=sci_arttext\&pid=S0100-67622013000600010 >. Acesso em: 10 maio 2015.

FERNANDES, L. L.; COELHO, A. B.; FERNANDEZ, E. A.; LIMA, J. E. Compensação e Incentivo à Proteção Ambiental: o caso do ICMS Ecológico em Minas Gerais. Revista de Economia e Sociologia Rural, Brasília, v. 49, n. 3, p. 521-544, jul/set 2011. Disponível em: <http://www.scielo.br/scielo. php?script=sci_arttext\&pid=S0103-20032011000300001 >. Acesso em: 10 set. 2017.

FRANCO, D. H.; FIGUEIREDO, P. J. M. Os impostos ambientais (taxação ambiental) no Mundo e no Brasil - O ICMS Ecológico como uma das opções de instrumentos econômicos para a defesa do meio ambiente no Brasil. Anuário da Produção Acadêmica Docente, Valinhos, v. 1, n. 1, p. 248258. 2007. Disponível em: <http://repositorio.pgsskroton.com.br/bitstream/123456789/1315/1/ Artigo\%2034.pdf>. Acesso em: 10 set. 2017.

FRENTE NACIONAL DOS PREFEITOS. Finanças dos municípios. Disponível em: <http://aequus. com.br/comparabrasil/>. Acesso em: 05 maio 2015.

FUNDAÇÃO JOÃO PINHEIRO. Lei Robin Hood. 2013. Disponível em: <http://www.fjp.mg.gov.br/ robinhood/index.php/leirobinhood/legislacao/lei1204 95>. Acesso em: 05 maio 2015. 
INSTITUTO BRASILEIRO DE GEOGRAFIA E ESTATíSTICA (IBGE). População. Disponível em: < http:// www.ibge.gov.br/home/mapa_site/mapa_site.php\#populacao>. Acesso em: 05 maio 2015.

JARDIM, M. H.; BURSZTYN, M. A. Pagamento por serviços ambientais na gestão de recursos hídricos: o caso de Extrema (MG). Engenharia Sanitária e Ambiental, Rio de Janeiro, v. 20, n. 3, p. 353 - 360 jul./set. 2015. Disponivel em: <http://www.scielo.br/pdf/esa/v20n3/1413-4152-esa-20-03-00353.pdf>. Acesso em: 10 set. 2017.

MASSOTE, V. A.; VIEIRA, N. M. Análise espacial da importância do ICMS Ecológico para os municípios de Minas Gerais no período de 2003 a 2012. In: Seminário da Economia Miniera. 16., 2014, Diamantina. Anais... Belo Horizonte: UFMG/Cedeplar, 2014. 1-23p. Disponível em: <http:// diamantina.cedeplar.ufmg.br/2014/site/arquivos/analise-espacial-da-importancia-do-icms-ecologico-para-os-municipios-de-minas-gerais.pdf>. Acesso em: 10 set. 2017.

MAXWELL, A. E. Principal component analysis. In . Multivariate analysis in behavioural research. London: Chapman and Hall, 1977. p. 39-45.

MAY, P. H.; LUSTOSA, M. C.; VINHA, V. (Orgs.). Economia e Meio Ambiente: Teoria e Prática. Rio de Janeiro: Elsevier, 2003.

MINAS GERAIS. Lei $\mathbf{n}^{\circ}$ 18.030, de 12 de janeiro de 2009. Disponível em: <http://www.fazenda.mg.gov.br/empresas/legislacao_tributaria/leis/2009//18030_2009.htm>. Acesso em: 05 maio 2015.

MONTE, M. A.; SILVA, M. L. da. Análise de repasse do ICMS Ecológico aos munícipios do estado de Minas Gerais. Revista Cerne, Lavras, v. 15, n. 4, p. 391-397, out./dez. 2009. Disponível em: $<$ http://ciflorestas.com.br/arquivos/doc_analise_gerais_12208.pdf>. Acesso em: 10 set. 2017.

OKSANEN, J.; BLANCHET, F. G.; FRIENDLY, M.; KINDT, R.; LEGENDRE, P.; MCGLINN, D.; MINCHIN, P. R.; O'HARA, R. B.; SIMPSON, G. L.; SOLYMOS, P.; STEVENS, M. H. H.; SZOECS, E.; WAGNER, H. Community Ecology Package. 2015. Disponível em: <http://cran.r-project.org/web/packages/vegan/ vegan.pdf >. Acesso em: 05 maio 2015.

PEARCE, D. W.; TURNER, R. K. The optimal level of pollution. In: (Ed.). Economic of natural resources and environment. Great Britain: British library, 1990. Chapter. 4, p. 61-67.

PINHEIRO, R. P.; SCHMIDT, P.; SANTOS, J. L. dos.; FERNANDES, L. A. Gestão dos recursos hídricos e o agronegócio: O problema da utilização do recurso hídrico e a ineficiência de mercado. In: ENCONTRO NACIONAL DE ENGENHARIA DE PRODUÇÃO, 30, 2010. São Carlos. Anais... São Carlos, ABEPRO. p. 1-14, 2010.

REZENDE, J. L. P.; OLIVEIRA, A. D. Análise econômica e social de projetos florestais. Viçosa: Universidade Federal de Viçosa, 2001. 389 p.

SECRETARIA DE ESTADO DE MEIO AMBIENTE E DESENVOLVIMENTO SUSTENTÁVEL - SEMAD. ICMS Ecológico. Disponível em: <http://www.meioambiente.mg.gov.br/icms-ecologico>. Acesso em: 04 ago. 2016. 
THE NATURE CONSERVANCY - TNC. ICMS Ecológico - Brasil. Disponível em: <http://www.icmsecologico.org. br/site/index.php?option=com_content\&view $=$ article\&id=54\&ltemid $=62>$. Acesso em: 05 maio 2015.

\section{Histórico editorial:}

Submetido em: 01/06/2016

Aceito em: 26/01/2017

\section{Como citar:}

ABNT

ROMERO, F. M. B.; SILVA, L. F. da; ISBAEX, C.; ALVES, E. B. B. M.; JACOVINE, L. A. G.; SILVA. M. L. da. O ICMS ecológico como instrumento econômico de melhorias ambientais e sociais em alguns municípios mineiros. Revista Agrogeoambiental, Pouso Alegre, v. 9, n. 3, p. 95-104, jul./set.

Doi: http://dx.doi.org/10.18406/2316-1817v9n320171008

\section{APA}

ROMERO, F. M. B., SILVA, L. F. da, ISBAEX, C., ALVES, E. B. B. M., JACOVINE, L. A. G. \& SILVA. M. L. da. (2017). O ICMS ecológico como instrumento econômico de melhorias ambientais e sociais em alguns municípios mineiros. Revista Agrogeoambiental, Pouso Alegre, 9 (3), 95-104.

Doi: http://dx.doi.org/10.18406/2316-1817v9n320171008

\section{ISO}

ROMERO, F. M. B.; SILVA, L. F. da; ISBAEX, C.; ALVES, E. B. B. M.; JACOVINE, L. A. G. e SILVA. M. L. da. O ICMS ecológico como instrumento econômico de melhorias ambientais e sociais em alguns municípios mineiros. Revista Agrogeoambiental, 2017, vol. 9, n. 3, pp. 95-104. Eissn 2316-1817.

Doi: http://dx.doi.org/10.18406/2316-1817v9n320171008

\section{VANCOUVER}

Romero FMB, Silva LF da, Isbaex C, Alves EBBM, Jacovine LAG, Silva ML da. O ICMS ecológico como instrumento econômico de melhorias ambientais e sociais em alguns municípios mineiros. Rev agrogeoambiental. 2017 jul/set; 9(3): 95-104.

Doi: http://dx.doi.org/10.18406/2316-1817v9n320171008 